\title{
Predictors of long term survival after surgical resection in carcinoma gall bladder
}

\author{
Gupta $V^{1}$, Kumar $S^{2}$, Rahul ${ }^{3}$, Chandra $A^{4}$ \\ ${ }^{1}$ Dr Vivek Gupta, Assistant Professor, Department of Organ Transplant, ${ }^{2}$ Dr Saket Kumar, Assistant Professor, \\ Department of Surgical Gastroenterology, ${ }^{3}$ Dr Rahul, Senior Resident, Department of Surgical Gastroenterology, \\ ${ }^{4}$ Dr Abhijit Chandra, Professor, Department of Surgical Gastroenterology, all authors are affiliated with King George's \\ Medical University, Lucknow (UP), India.
}

Address for Correspondence: Dr Vivek Gupta, Assistant Professor, Department of Organ Transplant, K.G.M.U. Lucknow, India. Email: vgkem@yahoo.com, vivekgupta@kgmcindia.edu

\begin{abstract}
Background-Carcinoma of the gallbladder is known to be associated with a dismal prognosis, with majority of patients presenting at late stages. Controversies still exist regarding the optimal management and the extent of surgery. MethodsThis study was done at a tertiary hospital in an endemic region for cancer gallbladder (north Indian Gangetic belt). All patients operated for cancer gallbladder between January 2006 and January 2011 were included, with details of clinical presentation, survival analysis and identification of prognostic factors. Results- Of the total 252 patients of carcinoma gall bladder identified during the study period, 71 patients were explored surgically. Curative resection was achieved in 27 patients with 10 long term survivors (disease free interval >3years). Nodal status, TNM stage and bilirubin levels affect survival. Majority of patients presented late in the course of disease (Mean of $\mathbf{4 4}$ days from the onset of symptoms). Conclusion-Majority patients with carcinoma gallbladder in our region have advanced unresectable disease. Bilirubin levels at presentation predict resectability. Nodal involvement implies a worse prognosis; however the extent of lymphadenectomy to be done is still debatable. Community screening programs and sensitization of local physicians for early referral can improve survival in endemic areas.
\end{abstract}

Keywords: Cancer, Gallbladder, Nodes, Bilirubin, Surgery

\section{Introduction}

Carcinoma of the gallbladder is known to be associated with an overall dismal prognosis [1-3]. Majority of patients are diagnosed at late stages of the disease resulting in poor surgical results [4-6]. Five year survival rates can range from 5 to 75 per cent [5-8] and the prognosis is stage dependent [9-11]. Patients with early gallbladder carcinoma may have survival rates greater than 70 per cent at 5 year [5, 9-11].

Despite increased understanding, controversies regarding the type of curative surgical treatment for each stage of the disease remain [12]. Reports suggest that aggressive resections like hepatopancreaticoduodenectomy can have a possibility of

Manuscript received $24^{\text {th }}$ October 2016

Reviewed: $4^{\text {th }}$ November 2016

Author Corrected: $15^{\text {th }}$ November 2016

Accepted for Publication $30^{\text {th }}$ November 2016 better outcome than usual in patients with advanced gallbladder cancer [13-17]; nevertheless advantages of radical surgery for cancer of the gallbladder still remain controversial.

A review of 4770 patients in Japan did not support any advantage for aggressive surgical resection and adjuvant chemotherapy [18].

Surgical morbidity and mortality rates continue to remain high after radical surgery $[19,20]$.

This study was under taken to analyse the clinical presentation and surgical outcomes of patients of cancer gallbladder at a tertiary care University hospital in north Indian Gangetic belt with a known high prevalence for carcinoma gall bladder [21]. 


\section{Methods}

This study was carried out at the Department of Surgical Gastroenterology, King George's Medical University, Lucknow, India. All patients diagnosed with gallbladder carcinoma from January 2006 to January 2011 were included in this study.

Relevant data was obtained from our prospectively maintained digital database as well as review of patient's records. Multiple parameters were analysed including clinical history, examination findings, complete blood count, coagulation profile, liver and renal function, radiological findings and interventions performed (Surgical, radiological and endoscopic).

As a part of routine workup all patients suspected to have carcinoma gall bladder underwent contrast enhanced CT scan of abdomen as well as abdominal ultrasound correlation to determine the extent of disease.

Further tests including MRI, PET scan and chest CT were done if required for staging or surgical planning. Radiological findings were recorded in detail, including the number and size of gall stones, gallbladder wall thickening, mass, contiguous or non contiguous spread, bile duct diameter, regional lymph nodes, ascites and intraperitoneal spread.

The cancers were classified by using the staging criteria of the American Joint Committee on Cancer 2010, $7^{\text {th }}$ edition $^{22}$. Tis, carcinoma in situ; TI, tumor invades the lamina propria or muscle layer; $\mathrm{T} 2$, tumor invades perimuscular connective tissue, with no extension beyond the serosa or into the liver; $\mathrm{T} 3$, tumor invades beyond the serosa into liver and/or one adjacent organ; $\mathrm{T} 4$, tumor involves hepatic artery or main portal vein or multiple extrahepatic organs; N0, Nodes absent; N1, involvement of nodes in the hepatoduodenal ligament and $\mathrm{N} 2$, nodes in the celiac axis, superior mesenteric axis or aortocaval region.

After pre-op work up patients were divided into operable and non-operable groups. The criterion for unresectability being: malignant lymphadenopathy along the celiac axis, superior mesenteric axis or aortocaval region, malignant ascites, multiple hepatic metastasis or distant metastasis. T4 lesion per se was not taken as suggestive of unresectability. Contrast enhanced CT scan abdomen was relied upon for the preoperative staging. If the intraoperative findings were found to be different from the CT findings and these were recorded and the procedure was modified accordingly.

Patients with obstructive jaundice selectively underwent preoperative biliary drainage procedure if major hepatic resection was planned or if serum bilirubin was more than $15 \mathrm{mg} / \mathrm{dl}$. Cholangitis was another indication for drainage. Percutaneous transhepatic biliary drainage or ERCP stent placement was done depending on the level of block. A biopsy was usually not performed unless in inoperable cases, before starting chemotherapy. A variety of resection procedures were applied, ranging from extended cholecystectomy, with or without common bile duct resection, extended right hepatectomy, gastric and colonic resections. Prophylactic resection of surrounding organs without apparent tumor invasion was not employed.

Regional lymphadenectomy was performed routinely which involved nodes in the hepatoduodenal ligament (N1) and nodes behind the head of the pancreas. Radical resection was abandoned when gallbladder carcinoma was found to be associated with multiple hepatic metastasis, peritoneal seeding, and celiac or para-aortic lymph node metastasis that could not be identified pre-operatively.

Patients in unresectable group were offered palliation of various sorts mainly chemotherapy. Biliary stenting or surgical bypass (segment 3 bypass, left duct bypass, hepaticcojejunostomy) with or without gastrojejunostomy were done to relieve jaundice or gastric outlet obstruction. Radiotherapy was not offered either as palliation or adjuvant therapy by our centre. Patients who refused any further treatment after counseling were offered effective pain relief and general palliative care. Follow-up data were obtained by reviewing hospital visits as well as telephonically. Survival was estimated by the Kaplan-Meier analysis. There was a minimum follow up of 5 years and a maximum of 10 years in this study.

Patients who survived for more than 3 years were compared with those who died of recurrent disease within 3 years to identify factors associated with long term survival. The prognosis was calculated stage wise. The differences among groups were tested by the log rank test, Chi square test and Mann Whitney U test as appropriate (SPSS-Software). 


\section{Results}

A total of 252 patients with a diagnosis of carcinoma gallbladder were identified from January 2006 to January 2011. Patients had a mean age of $48.7 \mathrm{yrs}$ and a female predominance $(76.1 \%, \mathrm{n}=192)$. The main physical findings at presentation seen in these patients were pain $(87.6 \% \mathrm{n}=221)$, gallbladder mass $(75.7 \%, \mathrm{n}=191)$, icterus $(42 \%, \mathrm{n}=106)$, anemia $(38.2 \%, \mathrm{n}=96)$ and ascites $(13.4 \%, \mathrm{n}=34)$. On radiological evaluation gallbladder wall thickening/irregularity with or without mass lesion was seen in $97.2 \%$ patients $(n=245)$, contiguous spread $(75 \%, n=189)$, non contiguous metastasis $(32 \%, \mathrm{n}=82)$, nodal involvement $(78.1 \%, \mathrm{n}=197)$, ascites $(23 \%, \mathrm{n}=58)$ and gall stones were present in $74.2 \%$ of patients $(\mathrm{n}=187)$. Mean duration of presenting to our institute after the onset of symptoms was 44 days.

Out of the 252 patients, majority $181(71.8 \%)$ were found to have advanced malignancy beyond any possibility of surgical cure based on the previously mentioned criterion for unresectability and were offered palliative or symptomatic treatment as chosen in consultation with the patient (CHART-1). Palliative treatment involved use of chemotherapy, biliary stenting or biliary bypass (seg. 3 bypass/ left duct drainage) and gastrojejunostomy for patients with gastric outlet obstruction. Effective pain relief in the form of celiac axis blocks was also considered. Thirty eight patients refused any further treatment after counseling and were offered pain relief and general palliative care.

Seventy one patients underwent surgical exploration with curative intent. TABLE 1 gives the details of the AJCC [22] staging of 71 patients explored. None of the patients in our series had a stage I or II lesion. Out of the 71 patients, it was possible to successfully remove the entire tumor and node burden (R0 resection) in 27 patients only. The rest 44 had either an unresectable disease on intra-operative evaluation, which was underestimated by radiology scans or the tumor involved the surgical margin as confirmed by biopsy (R1/R2 resection). Duodenum was the most common extrahepatic organ involved in the operated patients $(n=13)$. Two patients underwent duodenal sleeve resections; however hepatopancreatoduodenectomy as a means of curative resection was not employed in this series of patients. Gastrojejunostomy was performed as a palliation in 11 patients of duodenal involvement. 12 patients underwent a palliative surgical procedure to relieve jaundice (Segment 3/Left duct bypass). Biopsy alone was done in 11 patients (CHART1). The most common surgical procedure carried out was an extended cholecystectomy. The list of all procedures is shown in TABLE 2 . Three patients were referred to us after the pathologist identified the disease in the biopsy specimen (Incidental carcinoma gallbladder). CT was done to stage these patients in which one patient was found to have unresectable disease. Two patients underwent surgery however diagnostic laparoscopy revealed multiple peritoneal deposits in both patients and biopsy only was taken.

There were overall 2 mortalities in immediate postoperative phase out of 27 operated patients. Morbidity rate was $25.9 \%$ (7 patients) and included respiratory infections, biliary leak, liver abscess, wound dehiscence etc. In our series, we did not encounter any patient with stage I or II disease. At a follow up of three years post surgery, 3 out of 6 patients with Stage IIIA; 9 out of 14 patients with stage IIIB and 5 out of 7 patients with stage IVA had died of recurrence. At last follow up 4 patients had died due to unrelated cause while 4 were healthy till date. There was no death due to disease recurrence after three years of surgery. One of the long term survivors had a multivisceral resection (Extended cholecystectomy with colonic and abdominal parietal wall resection). Kaplan Meier and log rank test was applied for survival analysis. The median survival time was 1.79 years. Stage wise difference in survival based on AJCC stage (CHART 2) was not found significant in this study probably owing to the small no of patients $(\mathrm{n}=27)$.

8 patients had N0 disease with 5 long term survivors while 19 patients had N1 disease also with 5 long term survivors (Log rank test $\mathrm{p}=0.07$ ). This $\mathrm{p}$ value is close to significance and our experience also makes us believe that nodal involvement defines a subset of patients which has a worse prognosis (CHART 3). Difference in survival based on T staging did not reach significant level in this study. Fundal lesions even if advanced (T4) tend to involve colon and hence may be more resectable than the lesions situated in gall bladder neck involving hilar structures and duodenum. Pre operative bilirubin levels had a direct implication on prognosis and the difference in the mean bilirubin levels of patients between survivors and non survivors was significant $\mathrm{p}=0.02$. Age of the patient had no influence on the overall survival in this study ( $\mathrm{p}=0.717$ ). There was no significant difference noted between survivors and non survivors based on sex $(\mathrm{p}=0.59)$ or the presence or absence of gall stones $(\mathrm{p}=0.952)$; however it has been our keen observation that younger 
patients tend to have more aggressive disease. This hypothesis is still to be proved by larger trials. The two prognostic factors identified in our study were nodal status (AJCC $7^{\text {th }}$ edition) and the preoperative bilirubin levels.

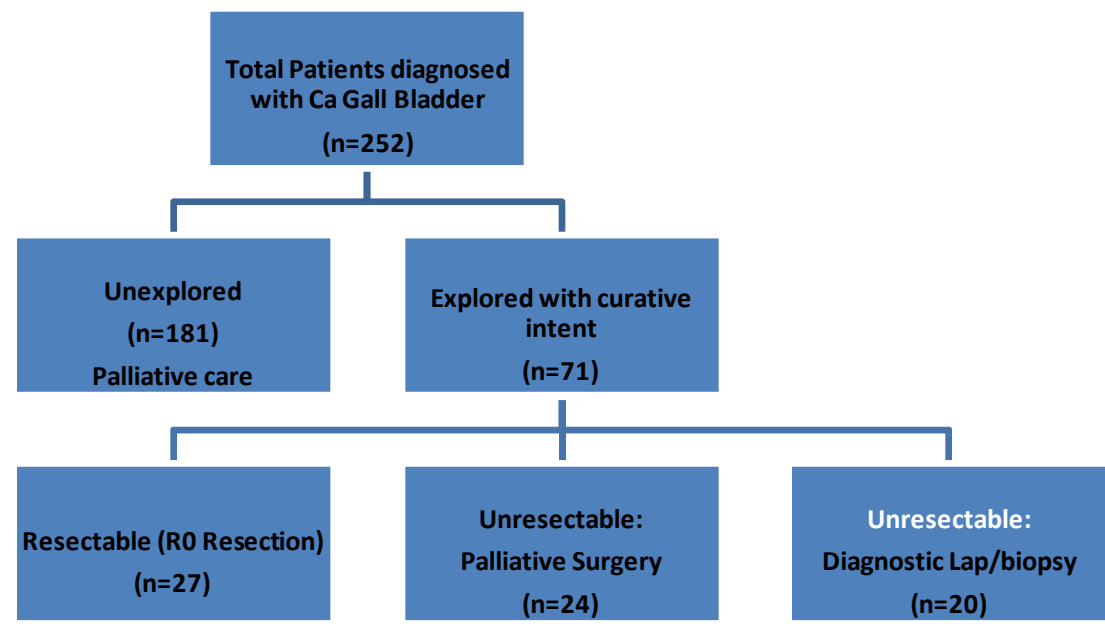

Chart-1: Outline of the management of 252 patients of carcinoma gall bladder enrolled in the study.

Table-1: AJCC staging ( $7^{\text {th }}$ edition) of 27 patients of carcinoma gall bladder who underwent successful R0 resection.

\begin{tabular}{|l|l|c|c|c|}
\hline Stage & TNM & Total Number & $\begin{array}{c}\text { Curative(R0) Resection } \\
\text { Achieved }\end{array}$ & $\begin{array}{c}\text { Survival > 3 } \\
\text { yrs }\end{array}$ \\
\hline 0 & Tis, N0, M0 & $\mathbf{0}$ & $\mathbf{0}$ & N/A \\
\hline I & T1, N0, M0 & $\mathbf{0}$ & $\mathbf{0}$ & N/A \\
\hline II & T2, N0, M0 & $\mathbf{0}$ & $\mathbf{0}$ & N/A \\
\hline III A & T3, N0, M0 & $\mathbf{6}$ & $\mathbf{6}$ & $\mathbf{3}$ \\
\hline III B & T 1-3, N1, M0 & $\mathbf{3}$ & $\mathbf{1 4}$ & $\mathbf{2}$ \\
\hline IVA & T4, N0, M0 & $\mathbf{8}$ & $\mathbf{5}$ & $\mathbf{0}$ \\
& T4, N1, MO & $\mathbf{1 5}$ & $\mathbf{0}$ & N/A \\
\hline IVB & Any T, N2, M0 (15) & $\mathbf{1 8}$ & $\mathbf{0}$ & N/A \\
& Any T, Any N, M1 (18) & $\mathbf{7 1}$ & $\mathbf{2 7}$ & $\mathbf{1 0}$ \\
\hline Total & & & & \\
\hline
\end{tabular}

Table-2: Details of surgical procedures performed on 71 patients of carcinoma gallbladder.

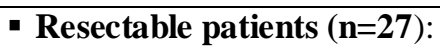

Extended cholecystectomy done in all patients along with

- Right Lobe Hepatectomy $(\mathrm{n}=3)$

- Excison of CBD ( $\mathrm{n}=9)$

- Multivisceral Resection ( $\mathrm{n}=6)$

Partial Gastrectomy and duodenal Wedge (1)

Colonic wedge resection (4)

Duodenal wedge resection (1)

- Unresectable Patients ( $=44)$ :

- Segment III bypass $(n=7)$

- Left duct bypass $(n=5)$

- Gastric bypass $(n=11)$

- Colonic bypass $(n=1)$ to relieve colonic obstruction.

- Inoperable - biopsy only $(\mathrm{n}=11)$ 


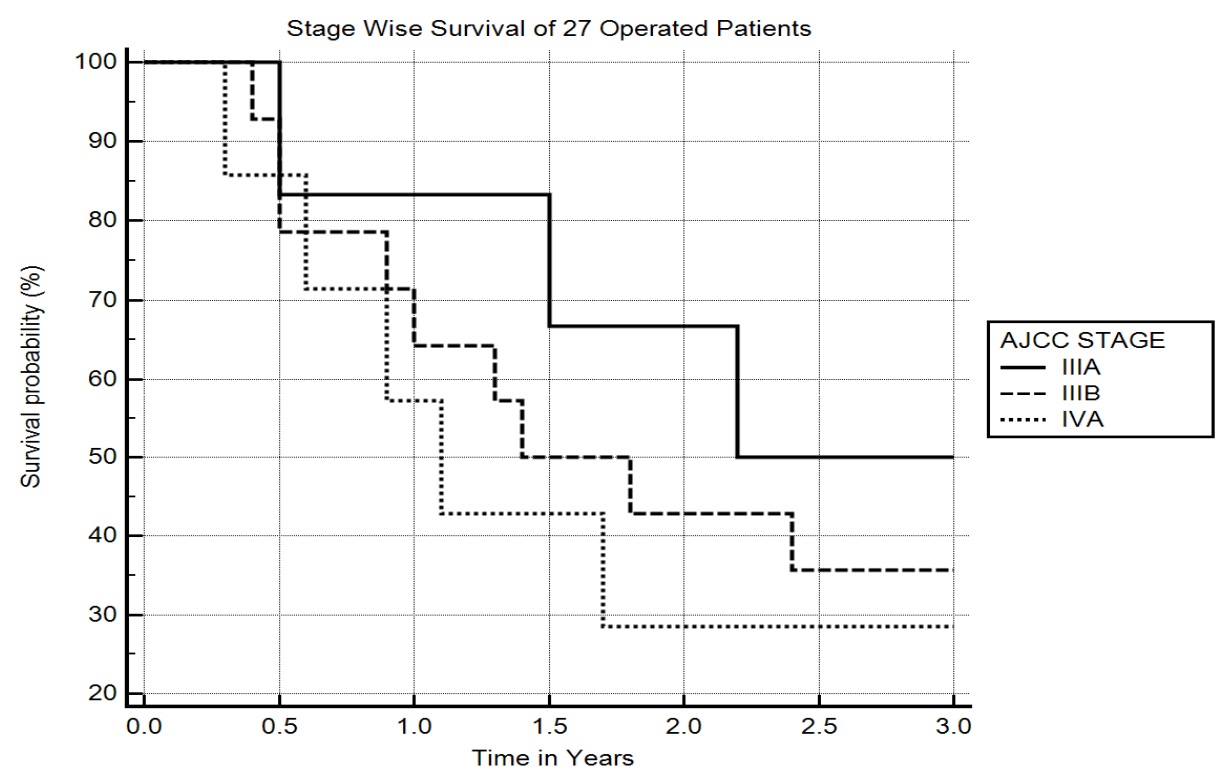

Chart-2: Kaplan Meier survival curve of 27 patients of surgically treated carcinoma gallbladder divided into stages based on $\mathrm{AJCC}-7^{\text {th }}$ edition

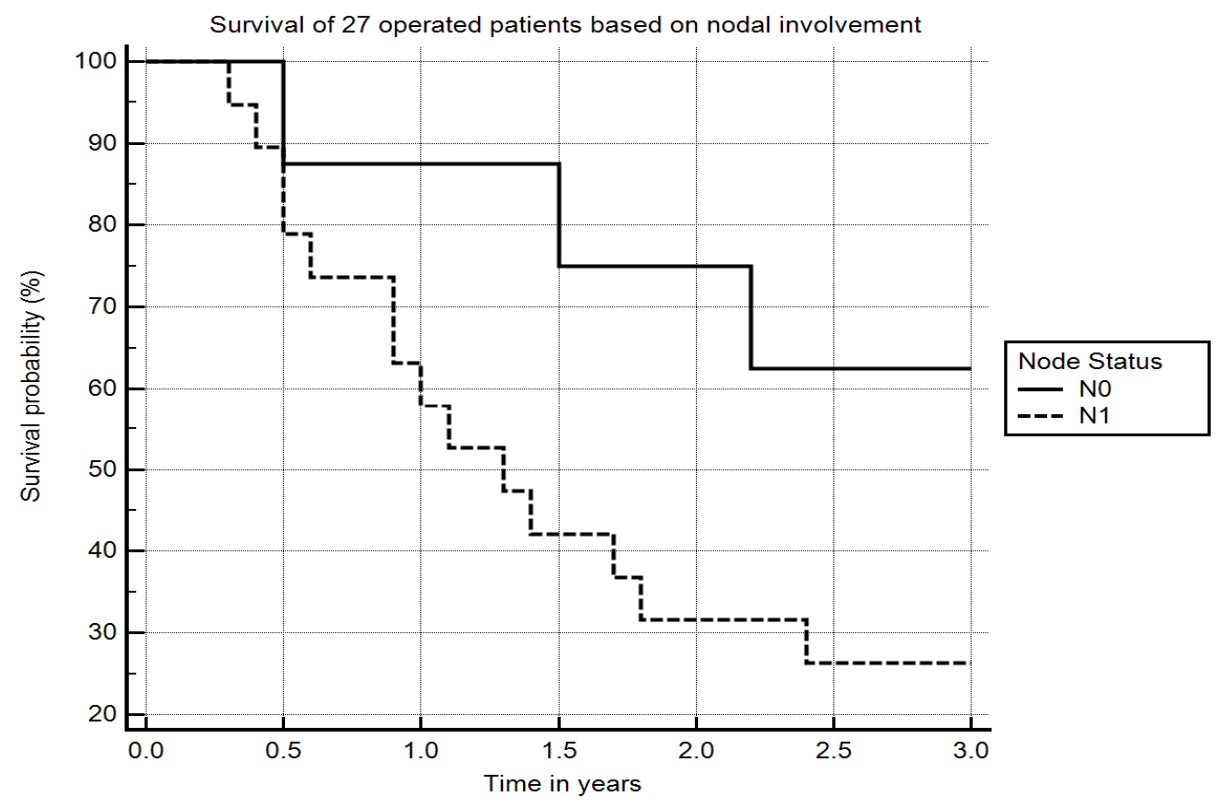

Chart-3: Kaplan Meier survival curve of 27 patients of surgically treated carcinoma gallbladder divided based on nodal status - $\mathrm{AJCC}-7^{\text {th }}$ edition

\section{Discussion}

The majority of patients with Gallbladder cancer in India have advanced unresectable disease. As noted in our study, only $28.2 \%$ patients had apparently resectable disease at initial presentation and finally curative resection could only be achieved in only $10.7 \%$ patients. Gallbladder malignancies are still diagnosed too late. In a review of 724 cases done by the French surgical association $77 \%$ of the patients had lesions beyond the possibility of any curative treatment [23]. This finding is not surprising when we consider that improved diagnostic imaging techniques cannot be applied until late in the disease course. Majority of patients with gallbladder carcinoma present at late stages of the disease resulting in a dismal overall prognosis [4-6, 24, 25]. More so this is especially true in our patient population from rural and Gangetic plains 
of India where people prefer alternate form of medication and are referred to us much later. Community screening programs and sensitization of local physicians for early referral can improve survival in endemic areas.

The prognosis of carcinoma of gallbladder has always been presented as dismal [1-8]. This is essentially the result of the slow and asymptomatic growth of the neoplasm that infiltrates the surrounding structures such as the portal vein and hepatic artery, making surgical treatment almost impossible. Several prognostic factors influence the clinical course of patients with gallbladder carcinoma: depth of tumor invasion, presence or absence of lymph node metastasis, histological type, surgical margin and TNM stage [26-28]. In our study nodal status was more important than the tumor size or conjugate spread in predicting the outcome.

The difference in the bilirubin levels between survivors and non survivors was statistically significant $(\mathrm{p}<0.02)$. Biliary tract carcinoma accompanied by obstructive jaundice is associated with increased surgical morbidity and mortality rates [13,19]. Higher pre operative bilirubin levels in this series of patients indicate a probability of advanced unresectable disease. Disease spectrum seems to be distributed into two patterns. An elderly age group $\geq 68$ years appears to have a more indolent and resectable disease with increased survival, while another group of younger patients (onset $\leq 50$ years) is characterized by more aggressive disease and poor survival outcomes. This difference needs to be statistically proven in further studies.

Satisfactory results can be achieved by simple or radical cholecystectomy in patients with stage I or II disease. Extensive surgery is not necessary in these patients [2931]. In contrast, the results of surgical treatment of stages III and IV gallbladder carcinoma have been reported to be extremely poor [2, 5]. Recently, the results have improved as a result of the introduction of extensive resection [32-34], including en bloc resection of involved organs especially by Japanese surgeons. The role of resectional surgery for $T_{3}$ and $T_{4}$ lesions is controversial as most authors report that patients with serosal involvement do not survive for 5 years even when major resections, including hepatectomy are performed [35-37].

Glenn and Hays had proposed the importance of lymph node dissection in surgery for GBC as early as 1954 .
The procedure was referred to as "skeletonization of the lesser Omentum.'[38] Radical resection with nodal dissection for GBC has been widely accepted with some surgeons including extensive dissection of lymph nodes in the peripancreatic portion, coeliac axis and paraaortic region $[11,17]$, however our experience is pessimistic about the radical procedure as once the $\mathrm{N} 2$ nodes are involved, radical surgery did not seem to improve outcomes. In our patient population the issues concerning the indications and limitations of lymph node dissection still need to be resolved. RO resection is required for the long-term survival of the patient with GBC. Direct invasion of the liver can be treated with liver resection. Wedge resection of the gallbladder bed (segment IV-V) can control limited liver invasion but extended right hepatic lobectomy has to be undertaken for a massive invasion of the liver or the invasion of the right main branches in the hepatic pedicle. A combined resection of the involved organs (pancreatoduodenectomy, colectomy, etc.) can achieve RO resection for $\mathrm{T} 3$ or $\mathrm{T} 4$ lesions. Combined resection and reconstruction of large blood vessels should be considered to obtain RO resection only in selected cases. A multidisciplinary approach, which includes neoadjuvant radiochemotherapy, may allow us to increase the rate of curative $\mathrm{RO}$ resection $[11,34]$.

As such there is still no consensus among the surgeons worldwide for a common protocol to manage carcinoma gallbladder. However all early stage cancers should be offered curative resectional surgery. Involvement of N2 node stations generally implies a grave prognosis.

The extent of lymphadenectomy is debatable, as is extensive surgery in the form of hepatopancreatoduodenectomy for advanced lesions. Despite best efforts the prognosis of cancer gallbladder remains grim in this part of the world.

\section{Conclusion}

Majority patients with carcinoma gallbladder in our region have advanced unresectable disease. Bilirubin levels at presentation predict resectability. Nodal involvement implies a worse prognosis however the extent of lymphadenectomy to be done is still debatable. Community screening programs and sensitization of local physicians for early referral can improve survival in endemic areas.

Funding: Nil, Conflict of interest: None initiated, Permission from IRB: Yes 


\section{References}

1. Wanebo HJ, Castle WN, Fechner RE. Is carcinoma of the gallbladder a curable lesion? Ann Surg 1982; 195: 624-31.

2. Donohue JH, Nagorney DM, Grant CS et al. Carcinoma of the gallbladder. Does radical resection improve outcome? Arch Surg 1990; 125: 237-41.

3. Batra Y, Pal S, Dutta U, Desai P et al. Gallbladder cancer in India: a dismal picture. J Gastroenterol Hepatol 2005 Feb; 20(2):309-14.

4. Gryko M, Dawidziuk T, Sawicka E et al. Cancer of the gallbladder - own experience. Prog Health Sci 2014 Jun;4(1):153-157.

5. Thorsten Oliver Goetze. Gallbladder carcinoma: Prognostic factors and therapeutic options. World J Gastroenterol 2015 Nov 21; 21(43): 12211-12217.

6. Gall FP, Kockerling F, Scheele J et al. Radical operations for carcinoma of the gallbladder: present status in Germany. World J Surg 1991; 15: 328-36.

7. Taner CB, Nagorney DM, Donohue JH. Surgical treatment of gallbladder cancer. J Gastrointest Surg 2004 Jan;8(1):83-9.

8. Grobmyer SR, Lieberman MD, Daly JM. Gallbladder cancer in the twentieth century: single institution's experience. World J Surg 2004 Jan;28(1):47-9.

9. Matsumoto Y, Fujii H, Aoyama H et al. Surgical treatment of primary carcinoma of the gallbladder based on the histologic analysis of 48 surgical specimens. Am J Surg 1992; 163: 239-45.

10. White K, Kraybill WG, Lopez MJ. Primary carcinoma of the gallbladder: TNM staging and prognosis. J Surg Oncol 1988; 39: 251-5.

11. Dwivedi AND, Jain S, Dixit R. Gall bladder carcinoma: Aggressive malignancy with protean locoregional and distant spread. World J Clin Cases. 2015 Mar; 3(3): 231-244.

12. Muratore A, Polastri R, Capussotti L. Radical surgery for gallbladder cancer: current options. Eur J Surg Oncol 2000 Aug;26(5):438-43.
13. Nimura $\mathrm{Y}$, Hayakawa N, Kamiya et al. Hepatopancreatoduodenectomy for advanced carcinoma of the biliary tract. Hepatogastroenterology 1991; 38: 170-5.

14. Nakamura S, Nishiyama R, Yokoi Y et al. Hepatopancreatoduodenectomy for advanced gallbladder carcinoma. Arch Surg 1994; 129: 625-9.

15. Yagi H, Shimazu M, Kawachi S et al. Retrospective analysis of outcome in 63 gallbladder carcinoma patients after radical resection. J Hepatobiliary Pancreat Surg 2006;13(6):530-6.

16. Foster JM, Hoshi H, Gibbs JF et al: Gallbladder cancer. Defining the indications for primary radical resection and radical re-resection. Ann Surg Oncol 2007 Feb;14(2):833-40.

17. Ishikawa T, Horimi T, Shima Yet al. Evaluation of aggressive surgical treatment for advanced carcinoma of the gallbladder. J Hepatobiliary Pancreat Surg 2003; 10(3):233-8.

18. Kayahara M, Nagakawa T. Recent trends of gallbladder cancer in Japan: an analysis of 4770 patients. Cancer 2007 Aug 1;110(3):572-80.

19. Tsukada K, Yoshida K, Aono $T$ et al. Major hepatectomy and pancreatoduodenec-tomy for advanced carcinoma of the biliary tract. Br J Surg 1994; 81: 108-10.

20. Meng H, Wang X, Fong Y et al. Outcomes of radical surgery for gallbladder cancer patients with lymphatic metastases. Jpn J Clin Oncol 2011 Aug;41(8):992-8

21. Dhir V, Mohandas KM. Epidemiology of digestive tract cancers in India IV. Gall bladder and pancreas. Indian J Gastroenterol 1999 Jan-Mar;18(1):24-8.

22. Edge SB, Byrd DR, Compton CC et al. AJCC cancer staging manual. 7thed.NewYork:Springer; 2010.

23. Pierre Cubertafond, Alain Gainant, Giovanni Cucchiaro. Surgical Treatment of 724 Carcinomas of the Gallbladder; Results of the French Surgical Association Survey. Annals of Surgery 1994 Vol. 219, No. 3, 275-280. 
24. Memon MA, Anwar S, Shiwani MH et al. Gallbladder carcinoma: a retrospective analysis of twenty-two years experience of a single teaching hospital. Int Semin Surg Oncol 2005 Mar 17;2(1):6.

25. Donohue JH. Present status of the diagnosis and treatment of gallbladder carcinoma. J Hepatobiliary Pancreat Surg 2001;8(6):530-4.

26. Buettner S, Margonis GA, Kim Y et al. Changing Odds of Survival Over Time among Patients Undergoing Surgical Resection of Gallbladder Carcinoma. Ann Surg Oncol 2016 Dec;23(13):44014409.

27. Henson DE, Albores-Saavedra J, Corle D. Carcinoma of the gallbladder. Histologic types, stage of disease, grade, and survival rates. Cancer 1992; 70: 1493-7.

28. D'Hondt $\mathrm{M}$, Lapointe $\mathrm{R}$, Benamira $\mathrm{Z}$ et al. Carcinoma of the gallbladder: patterns of presentation, prognostic factors and survival rate. An 11-year single centre experience..Eur J Surg Oncol 2013 Jun; 39 (6):548-53

29. Nigri G, Berardi G, Mattana C et al. Routine extrahepatic bile duct resection in gallbladder cancer patients without bile duct infiltration: A systematic review. Surgeon. 2016 Dec;14(6):337-344.

30. Aloia TA, Járufe N, Javle $M$ et al. Gallbladder cancer: expert consensus statement. HPB (Oxford). 2015 Aug;17(8):681-90.
31. Benoist S, Panis Y. Long-term results after curative resection for carcinoma of the gallbladder. Am J Surg 1998; 175: 118-22.

32. Bartlett DL, Fong Y, Fortner JG et al. Long-term results after resection for gall bladder cancer. Implications for staging and management. Ann Surg 1996; 224: 639-46.

33. Sikora SS, Singh RK. Surgical strategies in patients with gallbladder cancer: nihilism to optimism. J Surg Oncol. 2006 Jun 15;93(8):670-81.

34. Engineer R, Goel M, Chopra S et al. Neoadjuvant Chemoradiation Followed by Surgery for Locally Advanced Gallbladder Cancers: A New Paradigm. Ann Surg Oncol 2016 Sep;23(9):3009-15

35. Chijiiwa K, Yamaguchi K, Tanaka M. Clinicopathological differences between long term and short term postoperative survivors with advanced gall bladder carcinoma. World J Surg 1997; 21: 98-102.

36. He XD, Li JJ, Liu W et al. Surgical procedure determination based on tumor-node-metastasis staging of gallbladder cancer. World J Gastroenterol. 2015 Apr 21;21(15):4620-6.

37. Shirai Y, Sakata J, Wakai T et al. "Extended" radical cholecystectomy for gallbladder cancer: longterm outcomes, indications and limitations. World $\mathrm{J}$ Gastroenterol. 2012 Sep 14;18(34):4736-43.

38. Glenn F, Hays DM. The scope of radical surgery in the treatment of malignant tumors of the extrahepatic biliary tract. Surg Gynecol Obstet 1954; 99:529-541.

\section{How to cite this article?}

Gupta V, Kumar S, Rahul, Chandra A. Predictors of long term survival after surgical resection in carcinoma gall bladder. Int J Med Res Rev 2016;4(11):2046-2053.doi:10.17511/ijmrr. 2016.i11.25. 